\title{
MODAL-SPACE CONTROL OF A LINEAR MOTOR-DRIVEN GANTRY SYSTEM
}

\author{
C. Peukert ${ }^{1 *}$, P. Pöhlmann ${ }^{1}$, M. Merx ${ }^{1}$, J. Müller $^{1}$, S. Ihlenfeldt ${ }^{1,2}$ \\ ${ }^{1}$ Technische Universität Dresden, Faculty of Mechanical Science and Engineering, Institute of Mechatronic \\ Engineering, Department of Machine Tools Development and Adaptive Controls, Dresden, Germany \\ ${ }^{2}$ Fraunhofer Institute for Machine Tools and Forming Technology IWU, Dresden, Germany \\ *Corresponding author; e-mail: christoph.peukert@tu-dresden.de
}

\begin{abstract}
This paper presents the modal control applied to motion systems, in particular for machine tools. This control approach is particularly suitable for over-actuated systems that have more actuators than degrees of freedom. By using the modal approach, the parameterisation of the control loops is simplified since each control loop corresponds to a specific eigenmode.

A four-variable modal control of a linear motor-driven gantry system equipped with additional active damping devices is presented to achieve active vibration suppression. This approach is experimentally compared with a conventional control method that does not consider the eigenmodes of the system. The influence of the vibration controllers on the closed position loops is investigated.
\end{abstract}

\section{Keywords:}

Modal control; Active vibration control; Machine tool; Cascaded control; Linear motor; Inertial actuator

\section{INTRODUCTION}

Modal control is a control technique that aims at a complete diagonalisation of the system equations. Therefore, the modal control can be used to decouple the input-output behaviour of linear multivariable systems. The modal control for systems with lumped parameters was first indicated by [Rosenbrock 1962]. The modal control of systems with distributed parameters was introduced by [Murray-Lasso 1965] and [Gould 1966]. A detailed overview of the theory of modal control is given in [Simon 1968], [Porter 1972] and [Meirovitch 1990]. Between the 1970s and 1980s (see e.g. [Balas 1978] and [Meirovitch 1983b]) the modal space control was widely used for vibration suppression of flexible structures such as parts of spacecrafts. One of the most important control logics is the independent modal space control of the system's eigenmodes [Meirovitch 1983a]. Unlike other control logics, the modal control strategy has a well-defined physical meaning in the gain matrix definition [Serra 2013].

In general, controller tuning for multivariable systems, like machine tools, is not a straightforward task, especially when parallel acting drives and additional actuators are used. In this paper, the modal space control is applied to motion systems, in particular for machine tools. This approach represents a promising concept since the decoupled control loops are easier to design.

An essential goal in the development of machine tools is to increase productivity while maintaining or improving the accuracy of motion. For example, the increase in productivity can be achieved by increasing the dynamics of the machine tool. Linear motors are frequently used instead of conventional electromechanical drives for this purpose. In addition, lower moving masses [Kroll 2011], the use of redundant axis configurations [Schröder 2007] and higher drive forces can improve the dynamics of the moving components. For this reason, lightweight designs and multiple actuators per axis direction are increasingly used in machine tools. The so-called parallel drives are drive arrangements in which at least two drives are mechanically coupled via the machine structure and act on the same degree of freedom of movement [Peukert 2017]. A wellknown representative is the gantry type axis.

Highly dynamic motions cause both the moving structural assembly (e.g. slide) and the underlying machine structure (e.g. frame) to vibrate. High vibration amplitudes result especially for lightweight structures. Vibrations in machine tools lead to a deterioration in machining quality and must be avoided. In order to increase the dynamics while maintaining or improving the motion quality, measures to reduce or avoid vibrations are necessary. Methods for reducing the reaction force impacts are, for example, impulse decoupling [Drossel 2016], impulse compensation [Ihlenfeldt 2018] and Kinematically Coupled Force Compensation [Ihlenfeldt 2019]. An overview of methods for reducing the reaction force effect is given in [Großmann 2014]. Vibrations of the moving structure cannot be influenced by the aforementioned methods. However, such vibrations can be damped by the feed drives. In [Brecher 2013a], for example, drive-based vibration damping was used to increase the damping ratio of the first natural frequency by appropriate filtering of the measured position of the position control loop of a feed axis. In general, the reduction of the dynamic compliance of the machine tool at the Tool Centre Point (TCP) is aspired in order to increase the process stability and the chatter limit [Löser 2018]. This can be achieved by increasing the system's damping, e.g. by using passive or active damping systems. The tuned 
mass damper represents the most common passive auxiliary system. Because of its simplicity, it is widely used in machine tools. For example, [Yang 2010] applied multiple tuned mass dampers to increase chatter resistance of machine tool structures. However, active systems such as Active Damping Devices (ADDs) are preferred since they can achieve a higher power density than passive systems [Brecher 2013b]. In addition, damping actuators can be integrated relatively easily into existing machine tools without any design changes. It has been shown in numerous applications that such systems can significantly increase productivity and surface quality during milling (see e.g. [Lopez de Lacalle 2009]). Usually, the drives required for motion generation, as well as the additional, actuators are controlled independently, without considering mutual influences. The main drawback of this method is that the mechanical coupling is neglected and, thus, an interference between the control loops occurs, which makes the design of the controllers difficult [Belyaev 2017].

Most studies on modal control are limited to simple experimental set-ups such as beams [Wang 2002]. Exceptions are the modal state feedback control in combination with a modal state-observer of a flexible hydraulic manipulator [Resta 2010], the modal control of a 3 degree of freedom flexible parallel manipulator [Zhang 2014] and a model-based decoupling control for a dualdrive H-type gantry stage [Garcia-Herreros 2013]. However, the modal control approach offers advantages for the controller design of machine tools, which are demonstrated by means of the presented experimental study.

There are two preliminary studies for this contribution. Firstly, the modal control for vibration damping of an elastic structure (fixed gantry bridge) damped by two ADDs was investigated experimentally. Different control laws, including resonant vibration controllers, were analysed. Also, the modal space control was compared with the conventional control approach. It was possible to show that the modal decoupling allowed a simplified parameterisation of the control loops [Peukert 2019a]. Secondly, the modalspace control of a linear motor-driven gantry arrangement equipped with two ADDs was analysed through a simulation based process [Peukert 2019b]. With the modal control concept, a better closed loop behaviour can be achieved, while the disturbance behaviour at the TCP is the same as with the conventional approach.

In this paper, the aforementioned system is investigated experimentally. The system under consideration is decoupled by using the modal transformation, so that several modal control loops can be designed.

\section{MODAL-SPACE CONTROL FORMULATION}

With the use of a transformation between physical coordinates $\boldsymbol{q}$ and modal coordinates $\xi$ :

$$
q=\Phi \xi
$$

where $\boldsymbol{\Phi}$ is the matrix of the eigenvectors, the equation of motion of a mechanical system

$\boldsymbol{M} \ddot{\boldsymbol{q}}+\boldsymbol{D} \dot{\boldsymbol{q}}+\boldsymbol{K} \boldsymbol{q}=\boldsymbol{f}$

can be written in its modal form

$\ddot{\xi}+\Delta \dot{\xi}+\Lambda \xi=\Gamma^{-1} \tau$.

The system in Eq. (2) is defined by the matrices of inertia $\boldsymbol{M}$, damping $\boldsymbol{D}$ and stiffness $\boldsymbol{K}$ as well as the vector of torques and forces $\boldsymbol{f}$, which correspond to the coordinates $\boldsymbol{q}$. The vector $\boldsymbol{\tau}=\boldsymbol{\Phi}^{\mathrm{T}} \boldsymbol{f}$ represents the modal loads. The matrices of the modal inertias $\Gamma$ and eigenvalues $\Lambda$ have entries only on their main diagonal. In the case of a lightly damped system the matrix of the modal damping ratios $\boldsymbol{\Delta}$ can approximately be written as a diagonal matrix as well. Under these conditions, Eq. (3) consists of $N$ independent equations:

$\ddot{\xi}_{i}+2 \delta_{i} \dot{\xi}_{i}+\omega_{i}^{2} \xi_{i}=\gamma_{i}^{-1} \tau_{i}$, with $i=1 \ldots N$.

The aim of the modal approach is to control these independent modal systems. Therefore, the modal control laws are defined by

$\tau_{\mathrm{C}, i}=R_{\bmod , i}(\mathrm{j} \omega) \xi_{\mathrm{C}, i}$, with $i=1 \ldots n_{\mathrm{C}}$,

for a number $n_{\mathrm{C}}$ of modal coordinates $\xi_{\mathrm{C}}$. The modal coordinates are not physical coordinates and, therefore, cannot be measured directly. The determination of the modal coordinates can be achieved by means of observers [Resta 2010], spectral filters [Skidmore 1985] or modal filters [Meirovitch 1985]. In this paper, the calculation of $\xi_{\mathrm{C}}$ and the actuator forces $\boldsymbol{f}_{\mathrm{A}}$ is achieved by the modal filter $\boldsymbol{\Psi}^{\mathrm{T}}$ and the modal synthesizer $\boldsymbol{\Theta}$ (see [Zhang 2014])

$\xi_{\mathrm{C}}=\boldsymbol{\Psi}^{\mathrm{T}} \boldsymbol{q}_{\mathrm{S}}$ with $\boldsymbol{\Psi}^{\mathrm{T}}=\left(\boldsymbol{\Phi}_{\mathrm{CS}}\right)^{-1}$ and

$\boldsymbol{f}_{\mathrm{A}}=\boldsymbol{\Theta} \boldsymbol{\tau}_{\mathrm{C}}$ with $\boldsymbol{\Theta}=\left(\boldsymbol{\Phi}_{\mathrm{CA}}^{\mathrm{T}}\right)^{-1}$.

$\boldsymbol{\Phi}_{\mathrm{CS}}$ represents a submatrix of $\boldsymbol{\Phi}$ that contains only the columns of the controlled modes $\xi_{\mathrm{C}}$ and the rows of the measured coordinates $\boldsymbol{q}_{\mathrm{S}}$. In $\boldsymbol{\Phi}_{\mathrm{CA}}$ the rows corresponding to the forces $\boldsymbol{f}_{\mathrm{A}}$ are used. In order to invert the matrices in Eq. (6) and (7) there needs to be as many sensors and actuators as controlled modes. Generally, the sensors and actuators need to be placed in such a way that they are not located in the vibration nodes of the controlled eigenmodes. Since the full transformation $\boldsymbol{\Phi}$ is not used, mode shapes that are not controlled influence the estimates of $\xi_{C}$. Furthermore, the forces $f_{\mathrm{A}}$ also act on the uncontrolled modes. These effects are known as spillover [Meirovitch 1983c, Braghin 2012]. Spillover effects can be reduced if a sufficient number of sensors and actuators are used [Inman 2001]. It is recommended to position the actuators in the vibration nodes of the lowest uncontrolled modes and to place the sensors in the vibration nodes of the lowest unobserved modes [Wang 2002].

\section{EXPERIMENTAL SETUP}

The object of investigation is a linear motor-driven gantry system, which is moving in Y-direction (see Fig. 1). Two ADDs (ADD-45N, Micromega ${ }^{\circledR}$ ) are mounted on the movable $\mathrm{X}$-saddle. The physical principle and the corresponding transfer function of the inertial actuator are illustrated in Fig. 2, right. For this study, the X-saddle (approx. $15 \mathrm{~kg}$ ) is fixed in the centre of the gantry feed axis by using clamping elements. The different feedback controllers are implemented in the programmable logic controller in the TwinCAT ${ }^{\circledR}$ real time system. Several EtherCAT ${ }^{\circledR}$ terminals are used to enable the connection of the sensors/actuators. The communication between the sensors/actuators and the control unit is realised by an EtherCAT ${ }^{\circledR}$ fieldbus. A numerical model was developed in order to conduct simulative investigations regarding suitable control concepts, optimal sensor/actuator placements and was also used for the preliminary design of the components of the test rig [Peukert 2019b]. The test rig is illustrated in Fig. 1. $y_{\mathrm{le}, 1}$ and $y_{\mathrm{le}, 2}$ are the positions, measured with the linear encoders. $\ddot{y}_{\mathrm{acc}, 1}, \ddot{y}_{\mathrm{acc}, 2}, \ddot{y}_{\mathrm{acc}, 3}$ and $\ddot{y}_{\mathrm{acc}, 4}$ are the signals measured by the accelerometers. 


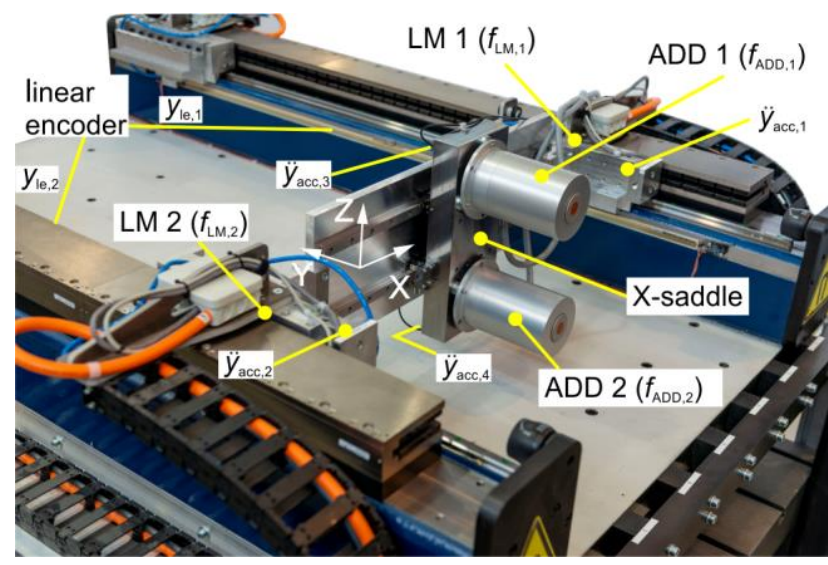

Fig. 1: Linear motor-driven gantry system with two ADDs.

In order to excite the modes in the $X-Y$-plane, the system is excited by an impulse hammer inclined $60^{\circ}$ to the $Y$-axis as can be seen in Fig. 2, left. An additional accelerometer is used to measure the acceleration $\ddot{q}_{\text {Imp }}$ in the same direction as the force $f_{\text {Imp }}$ is applied. A frequency analyser PULSE Modal 3560C (Brüel \& Kjaer) is used to calculate the frequency response functions (FRFs).

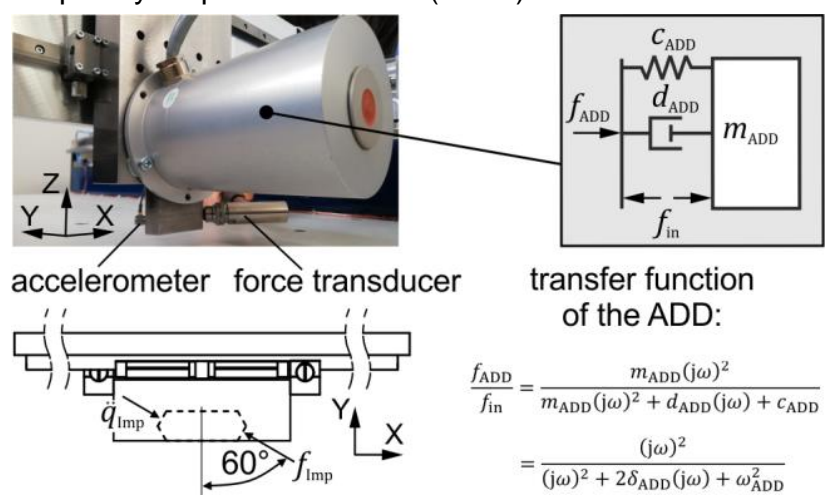

Fig. 2: Location and direction of the excitation (left) and model of the inertial actuator (right).

Fig. 3 shows the open loop FRFs and the target mode shapes of the analysed gantry system. The first eigenmode corresponds to the rigid body motion of the gantry bridge.

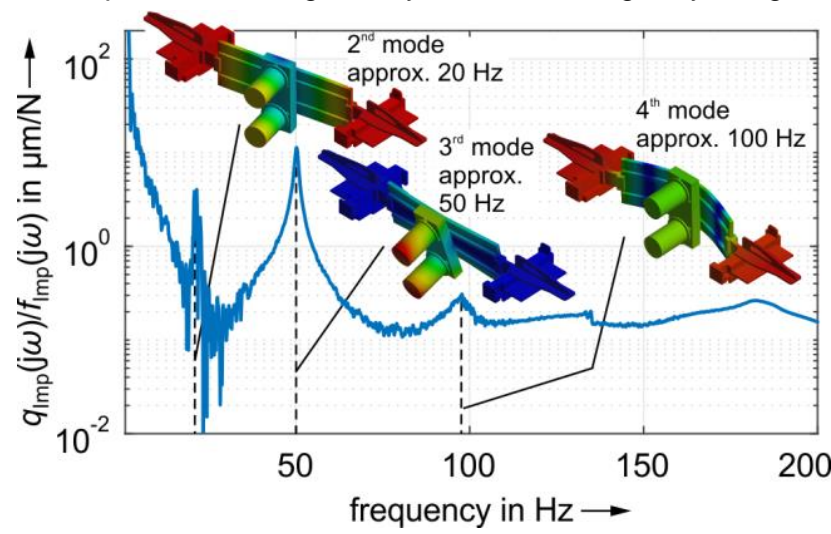

Fig. 3: Open loop FRF and the mode shapes of interest.

\section{EXPERIMENTAL ANALYSIS OF THE LINEAR MOTOR-DRIVEN GANTRY SYSTEM}

Firstly, the dynamic behaviour of the gantry system needs to be analysed. For this purpose, the system is excited by each of the four actuators, whereby pseudo random binary sequences (PRBS) are used as input force values. A low bandwidth motion controller keeps the gantry-stage in continuous motion to avoid issues arising from the static friction of the guiding system. With the acceleration measurement of the four coordinates (compare Eq. 8), the matrix $\boldsymbol{G}(\mathrm{j} \omega)$, which describes the systems FRFs is identified:

$\boldsymbol{y}=\left(\begin{array}{l}y_{\mathrm{acc}, 1} \\ y_{\mathrm{acc}, 2} \\ y_{\mathrm{acc}, 3} \\ y_{\mathrm{acc}, 4}\end{array}\right)=\underbrace{\left(\begin{array}{ccc}G_{11}(\mathrm{j} \omega) & \cdots & G_{14}(\mathrm{j} \omega) \\ \vdots & \ddots & \vdots \\ G_{41}(\mathrm{j} \omega) & \cdots & G_{44}(\mathrm{j} \omega)\end{array}\right)}_{\boldsymbol{G}(\mathrm{j} \omega)} \cdot\left(\begin{array}{c}f_{\mathrm{LM}, 1} \\ f_{\mathrm{LM}, 2} \\ f_{\mathrm{ADD}, 1} \\ f_{\mathrm{ADD}, 2}\end{array}\right)$

The amplitudes of the four FRFs of the main diagonal of $\boldsymbol{G}(\mathrm{j} \omega)$ are shown in Fig 4 (blue compliance curves). The peak of the fourth mode (approx. $100 \mathrm{~Hz}$ ) appears in each diagram, whereas the peak of the second mode (approx. $20 \mathrm{~Hz})$ occurs in $G_{11}(\mathrm{j} \omega)$ and $G_{22}(\mathrm{j} \omega)$ only, since the corresponding mode shape cannot be detected with the third and fourth accelerometer. On the other hand, the third mode (approx. $50 \mathrm{~Hz}$ ) can be found in $G_{33}(\mathrm{j} \omega)$ and $G_{44}(\mathrm{j} \omega)$ but not in $G_{11}(\mathrm{j} \omega)$ and $G_{22}(\mathrm{j} \omega)$. Therefore, the third mode cannot be excited with the linear motors.

\subsection{Modal decomposition of the system}

In order to control the modes of interest independently, the modal control loops should not affect each other. Therefore, the modes in the modal transfer functions of $\boldsymbol{G}_{\text {mod }}$ (compare Eq. (9)), need to be sufficiently separated. The goal of the separation is to have only one resonance peak per modal FRF $G_{\text {mod,ii }}(\mathrm{j} \omega)$. In order to design the modal system, the modal filter and the modal synthesizer need to be determined. With the matrix of the system's transfer functions $\boldsymbol{G}(\mathrm{j} \omega)$ the matrices $\boldsymbol{\Phi}_{\mathrm{CS}}$ and $\boldsymbol{\Phi}_{\mathrm{CA}}$, can be found. Since the actuators and sensors are nearly collocated, these matrices are approximately equal. With Eq. (6) and (7), the modal transformation of the system is obtained:

$\left(\begin{array}{c}\xi_{\mathrm{C}, 1} \\ \vdots \\ \xi_{\mathrm{C}, 4}\end{array}\right)=\underbrace{\boldsymbol{\Psi}^{\mathrm{T}} \cdot \boldsymbol{G}(\mathrm{j} \omega) \cdot \boldsymbol{\Theta}}_{\boldsymbol{G}_{\bmod }(\mathrm{j} \omega)} \cdot\left(\begin{array}{c}\tau_{\mathrm{C}, 1} \\ \vdots \\ \tau_{\mathrm{C}, 4}\end{array}\right)$.

The quality of the mode decomposition is verified by measuring the frequency response characteristics of the system, which correspond to each of the modal control loops. The main diagonal entries of the modal FRF matrix $\boldsymbol{G}_{\text {mod }}(\mathrm{j} \omega)$ are illustrated in Fig. 4 as well. Here, the first four modes are decoupled by means of the modal transformations. This can be seen by the corresponding peaks, which only appear in one of the main diagonal elements (red curves in Fig. 4). $G_{\bmod , 11}(\mathrm{j} \omega)$ shows a behaviour comparable to that of a second-order integrator, because the first mode represents the rigid body motion. The modes are separated efficiently, and the mutual influence of the modal loops is rather small. However, the truncated modes also appear in the FRFs of $\boldsymbol{G}_{\bmod }(\mathrm{j} \omega)$ as shown in Fig. 4 bottom, left. This effect corresponds to the aforementioned spillover problem. 

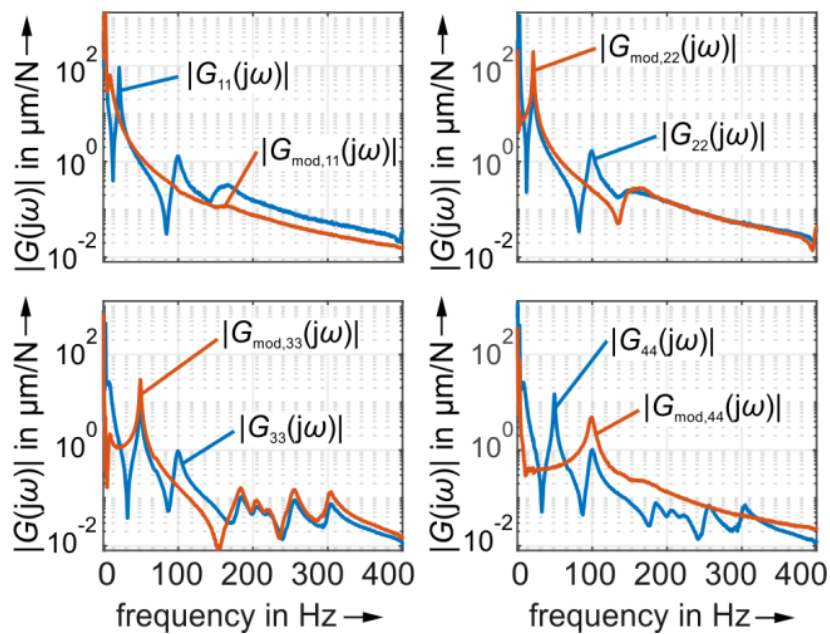

Fig. 4: Comparison of the main diagonal entries of the FRF-matrices, indicated in Eq. 8 and 9.

\subsection{Approaches for active damping control}

Fig. 5 shows a conventional control approach, where two cascaded position and velocity controllers (CPV) for both linear motors (LMs), as well as two additional controllers $R_{\text {loc }, i}$ for both ADDs, are used. The velocity loop consists of a PI-controller, whereas a P-controller is used in the position loop. Since each of the four controllers utilises an almost collocated actuator/sensor pair, this concept is called local control (LC) in this paper.

The modal control approach (MC) is displayed in Fig. 6. In this case, four acceleration signals are used and the transformation into modal coordinates $\ddot{\xi}_{\mathrm{C}, i}$ is achieved. This allows four modal control laws $R_{\text {mod, } i}$, whose outputs $\tau_{\mathrm{C}, i}$ are used to calculate the actuator forces, to be defined. The force values for the first two actuators are added to the values calculated by the CPV controllers.

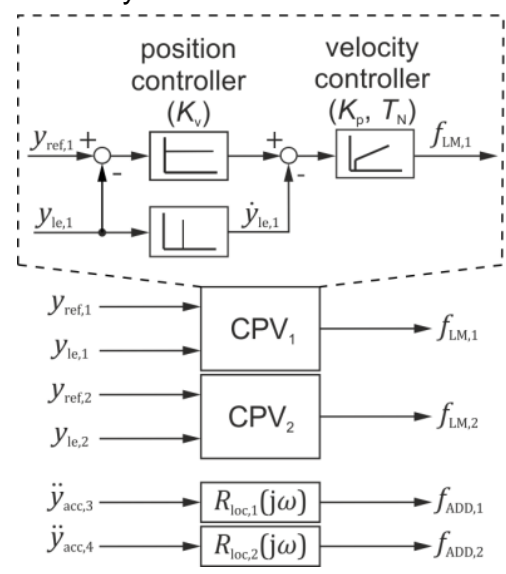

Fig. 5: Local control concept (LC).

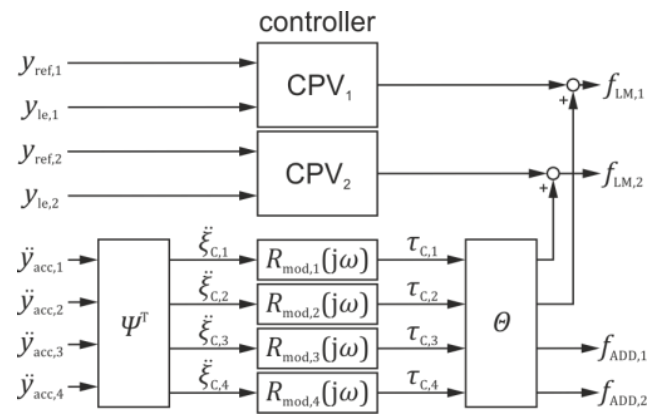

Fig. 6: Modal control concept (MC).
The next step is to define the local and modal transfer functions $R(\mathrm{j} \omega)$, which represent the control laws for active damping. Since the actuators and sensors are nearly collocated, a force value that has a phase shift of $180^{\circ}$ relative to the velocity is necessary to reduce vibration of a specific frequency [Gazzulani 2012]. Because accelerations are measured, an integrator (I) with a negative sign fulfils this demand. Therefore, the gain of the controller rolls off for higher frequencies. The complete control law, which is applied for both the local and modal approach, is given by:

$R(\mathrm{j} \omega)=\underbrace{-k}_{\text {gain }} \cdot \underbrace{\frac{1}{j \omega}}_{\mathrm{I}} \cdot \underbrace{\frac{\mathrm{j} \omega}{\left(j \omega+\omega_{\mathrm{H}}\right.}}_{\mathrm{HP}} \cdot \underbrace{\left(\mathrm{j} \omega+\omega_{1}\right)}_{\mathrm{PL}} \frac{-k\left(\mathrm{j} \omega+\omega_{1}\right)}{\left(\mathrm{j} \omega+\omega_{2}\right)}=\frac{-}{\left(\mathrm{j} \omega+\omega_{\mathrm{H}}\right)\left(\mathrm{j} \omega+\omega_{2}\right)}$.

An additional high-pass filter (HP) is used, which avoids issues at low frequencies arising due to the integration. The objective of the phase-lead compensator $(\mathrm{PL})$ is to get a higher phase margin and therefore to achieve a good control performance in a wider range of frequencies. The chosen control law forms a band-limited integrator, where the eigenfrequencies of the system do not have to be known exactly. The control law formulated in Eq. 10 is therefore insensitive to small parameter variations. This is advantageous for a practical application in machine tools, as the natural frequencies usually change depending on the mechanical configuration. However, when the eigenfrequencies of the system change in a wider range, the stability is not guaranteed. In addition, a variation of the mode shapes of the system requires the adaptation of the modal filter and the modal synthesizer in order to achieve a good separation of the modal control loops. However, this aspect is not addressed in this paper. The control parameters that are used in the remainder of this paper are listed in Tab. 1.

The frequency response functions given in Fig. 4 play an important role in the process of the control design, because they represent the systems behaviour from the "view" of the controllers. The local controllers $R_{\mathrm{loc}, 1}$ and $R_{\mathrm{loc}, 2}$, for example, are set to match $G_{33}(\mathrm{j} \omega)$ and $G_{44}(\mathrm{j} \omega)$. By contrast, the modal controllers $R_{\text {mod, } i}$ match the corresponding modal transfer function $G_{\bmod , i i}(\mathrm{j} \omega)$. Since only one of the considered eigenmodes appears in each of the modal transfer functions of the system, it is possible to find individual parameter sets for the modal controllers. In the case of the local approach, all eigenmodes appearing in $G_{33}(\mathrm{j} \omega)$ and $G_{44}(\mathrm{j} \omega)$ have to be taken into account.

\section{Control design with frequency response method}

An issue of the control approaches described in this paper is that the transfer functions of the controllers and the corresponding parameters have to be defined. For this purpose, experiments can be utilised. However, this would lead to an increased measuring effort. Another way is the use of a simulation model. This requires an accurate system knowledge to get representative results, and inaccuracies due to approximations are unavoidable.

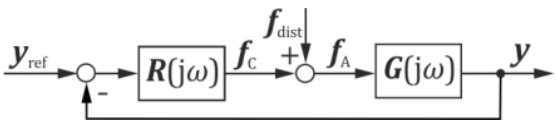

Fig. 7: Simplified control loop.

For the investigations in this work, the experimental identified matrix of FRFs of the system given in Eq. (8) is used. Each element $G_{i k}(\mathrm{j} \omega)$ of $\boldsymbol{G}(\mathrm{j} \omega)$ corresponds to a list of complex values for a discrete number of frequencies $2 \pi \omega_{k}(k=1 \ldots N)$. Fig. 7 shows a simple model of the closed control loop. The output $\boldsymbol{y}$ can be calculated by: 


$$
\begin{aligned}
& \boldsymbol{y}=\boldsymbol{G} \cdot\left(\boldsymbol{f}_{\mathrm{C}}+\boldsymbol{f}_{\mathrm{dist}}\right)=\boldsymbol{G R} \cdot\left(\boldsymbol{y}_{\mathrm{ref}}-\boldsymbol{y}\right)+\boldsymbol{G} \cdot \boldsymbol{f}_{\mathrm{dist}}, \\
& \Rightarrow \boldsymbol{y}=(\boldsymbol{G R}+\mathbf{E})^{-1} \boldsymbol{G R} \cdot \boldsymbol{y}_{\mathrm{ref}}+\underbrace{(\boldsymbol{G} \boldsymbol{R}+\mathbf{E})^{-1} \boldsymbol{G}}_{\boldsymbol{G}_{\mathrm{dist}}} \cdot \boldsymbol{f}_{\mathrm{dist}},
\end{aligned}
$$

where $\mathbf{E}$ is the identity matrix. Therefore, $(\boldsymbol{G} \boldsymbol{R}+\mathbf{E})^{-1} \boldsymbol{G R}$ represent the closed loop transfer function and $\boldsymbol{G}_{\text {dist }}$ the system's response behaviour caused by a disturbance $\boldsymbol{f}_{\text {dist }}$. In the case of the damping control, for example, $\boldsymbol{G}_{\text {dist }}$ is important because the aim is to improve the dynamic stiffness of the system. With a given matrix of controller transfer functions $\boldsymbol{R}$ and the measured open-loop-system $\boldsymbol{G}, \boldsymbol{G}_{\text {dist }}$ can be calculated with Eq. (12) for the discrete frequencies $2 \pi \omega_{k}$. In Fig. 8 the measured open loop FRF $G_{44}$ and the calculated, as well as the measured, FRFs $G_{\text {dist,44 }}$ for modal control are displayed as an example.

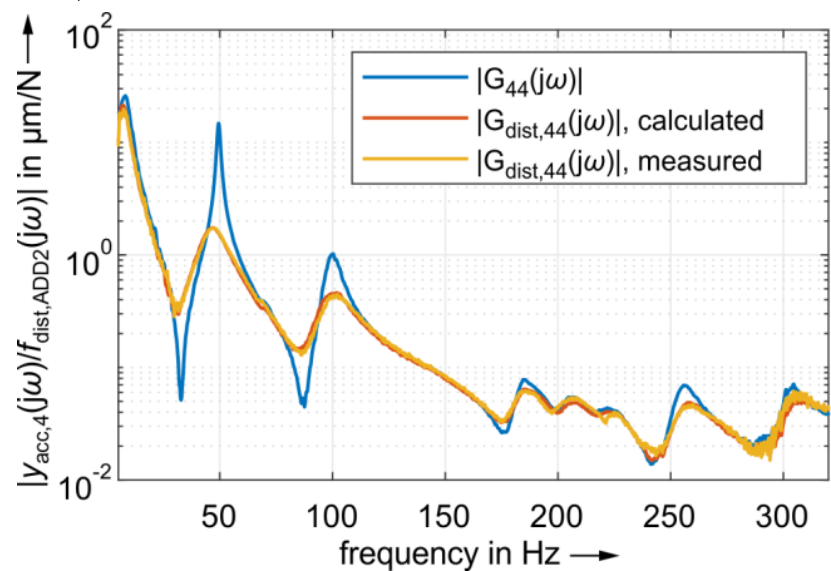

Fig. 8: Measured open loop FRFs and the calculated as well as the measured modal closed loop control FRFs.

The modal damping control with the parameters given in Tab. 1 is used here. The diagram shows a good conformity between the calculated and the measured curve. Therefore, the calculation in Eq. (12) based on the knowledge of the open loop FRFs $\boldsymbol{G}$ is an efficient method to estimate the closed loop behaviour. It allows for an examination of a wide variety of parameters and the finding of an appropriate solution. Optimisation algorithms are possible as well. In this study, frequency response design methods (see e.g. [Dorf 2011]) are applied in order to determine the structure (see Eq. (10)) and the parameters of the controllers. The parameters are analysed with the aforementioned method and are changed iteratively. The final control design chosen is then verified by experiments.

Tab. 1: Parameters of the local and modal control loops.

\begin{tabular}{ccccc}
\hline controller & $\boldsymbol{k} /\left(\mathbf{N s}^{2} / \mathbf{m}\right)$ & $\omega_{\mathrm{H}} / \mathbf{H z}$ & $\boldsymbol{\omega}_{\mathbf{1}} / \mathbf{H z}$ & $\boldsymbol{\omega}_{2} / \mathbf{H z}$ \\
\hline$R_{\mathrm{mod}, 1}$ & 200 & $10 \cdot 2 \pi$ & $200 \cdot 2 \pi$ & $8000 \cdot 2 \pi$ \\
$R_{\mathrm{mod}, 2}$ & 400 & $10 \cdot 2 \pi$ & $100 \cdot 2 \pi$ & $500 \cdot 2 \pi$ \\
$R_{\mathrm{mod}, 3}$ & 1000 & $20 \cdot 2 \pi$ & $100 \cdot 2 \pi$ & $500 \cdot 2 \pi$ \\
$R_{\mathrm{mod}, 4}$ & 500 & $40 \cdot 2 \pi$ & $200 \cdot 2 \pi$ & $1000 \cdot 2 \pi$ \\
$R_{\mathrm{loc}, 1}$ & 1000 & $20 \cdot 2 \pi$ & $100 \cdot 2 \pi$ & $500 \cdot 2 \pi$ \\
$R_{\mathrm{loc}, 2}$ & 1000 & $20 \cdot 2 \pi$ & $100 \cdot 2 \pi$ & $500 \cdot 2 \pi$ \\
\hline
\end{tabular}

\subsection{Experimental analysis of the active damping control}

In order to examine the dynamic behaviour of the local and modal control strategy for different controller gains, each of the controllers is investigated individually. For this analysis, the CPVs of the linear motors are deactivated. The system is excited by an impulse hammer, as illustrated in Fig. 2 , left.

Both of the local controllers act on mode three and four, resulting in increased damping ratios of the corresponding peaks at approximately 50 and $100 \mathrm{~Hz}$. The FRFs for the local controllers are very similar and therefore only the frequency behaviour of one local controller is depicted here. As an example, Fig. 9 shows the FRF of the system when only the second local controller $R_{\text {loc }, 2}$ is active. The parameters given in Tab. 1 are used, except the gain $k_{\mathrm{loc}, 2}$, which is varied in this experiment. The second eigenmode (approx. $20 \mathrm{~Hz}$ ) is not affected because of the location of the ADDs. As mentioned in Section 4.1, this mode does not appear in $G_{44}(\mathrm{j} \omega)$ and for this reason, the force output of the local controllers $R_{\mathrm{loc}, 1}$ and $R_{\mathrm{loc}, 2}$ cannot influence it. Each local controller may have a high gain value. However, when activating both local controllers, stability problems occur because of the mutual influence of the actuators (compare [Peukert 2019a]).

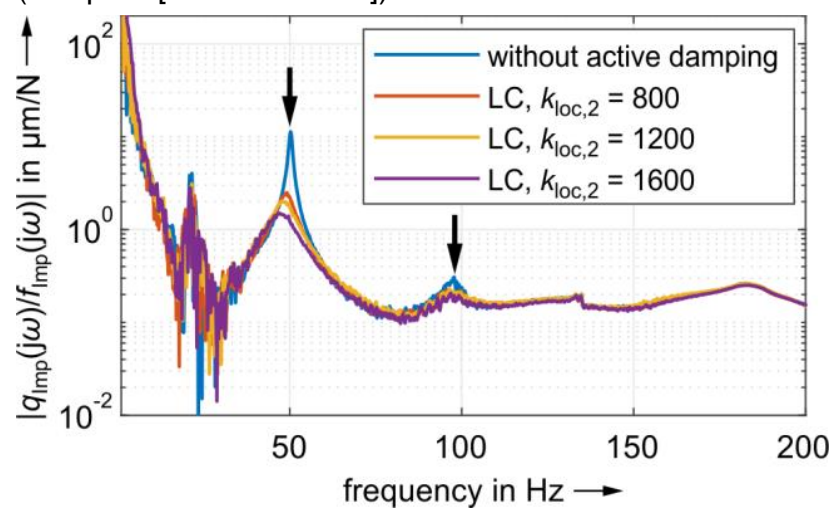

Fig. 9: Measured FRFs for different gains of the second local controller (first local controller inactive).

The experiment is repeated for the modal approach. In Fig. 10 the variation of the gain of the modal controller $k_{m o d, 2}$ is shown. It is evident that only the damping ratio of the second natural frequency has changed.

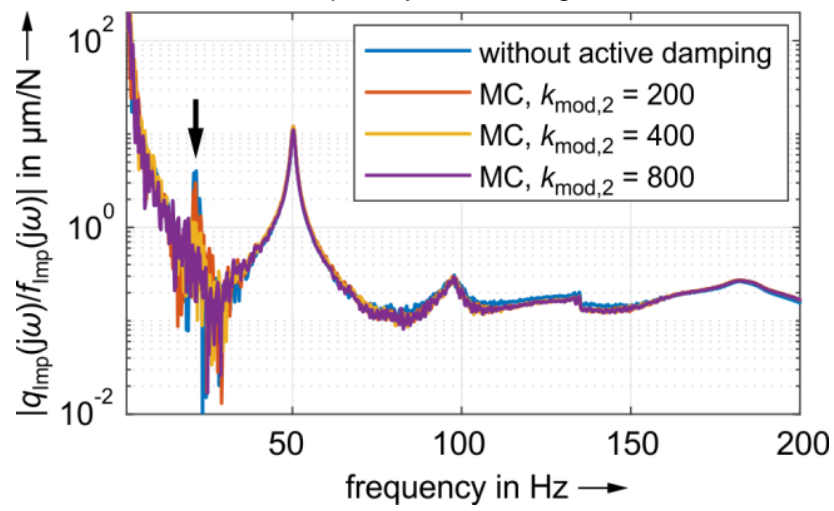

Fig. 10: Measured FRFs for different gains of the second modal controller (all other modal controllers inactive).

Fig. 11 depicts the FRFs whereby the gain $k_{\text {mod, } 3}$ is varied. In this case, only the third mode is affected. Conversely, the fourth modal controller increases only the damping ratio of the eigenfrequency at approx. $100 \mathrm{~Hz}$, which is illustrated in Fig. 12. In conclusion, it is shown that each modal controller is acting on only one of the system's eigenmodes and does not affect the others. This proofs the successful separation discussed in Section 4.1. The advantage of this effect is that the control laws can be designed for each eigenmode individually, which simplifies the whole controller parameterisation. 


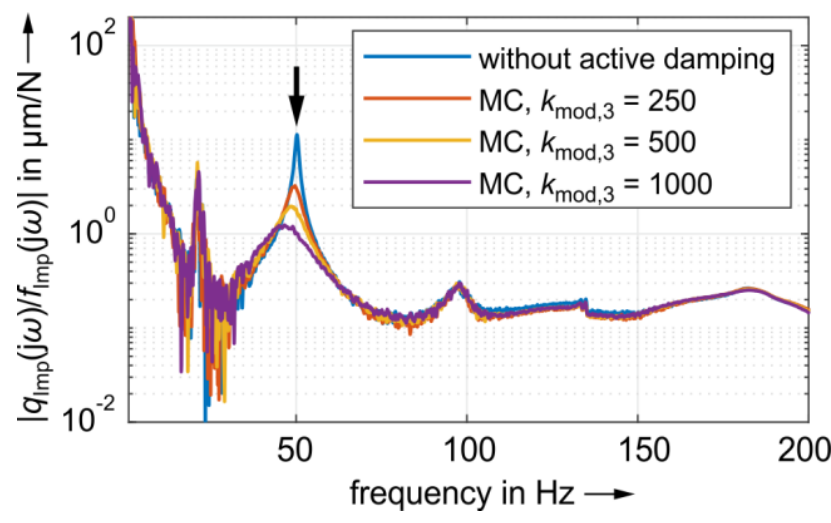

Fig. 11: Measured FRFs for different gains of the third modal controller (all other modal controllers inactive).

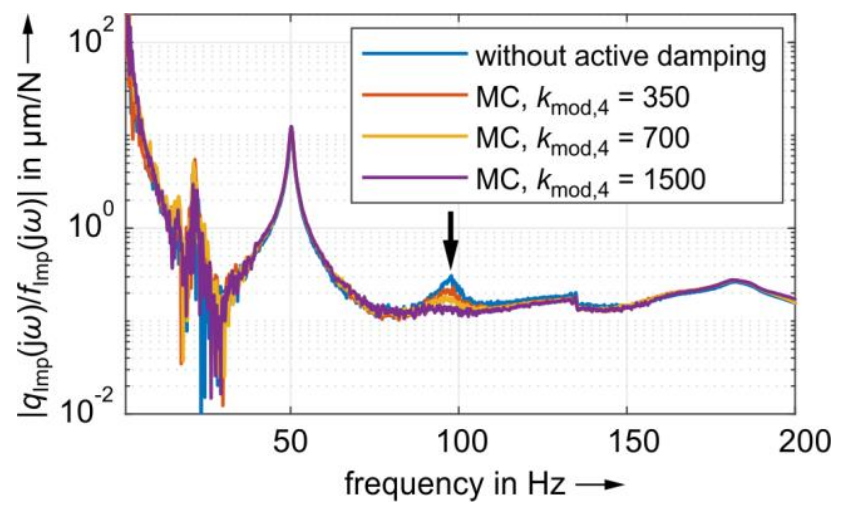

Fig. 12: Measured FRFs for different gains of the fourth modal controller (all other modal controllers inactive).

\subsection{Reference and disturbance behaviour of the gantry-arrangement}

In the next step, the influence of the additional damping controllers on the closed loop FRFs of the feed drives is investigated. For each linear motor, a CPV is used. For each control strategy, the same values are set in the velocity controller $\left(K_{\mathrm{P}}=2480 \mathrm{~N} /(\mathrm{m} / \mathrm{s}), \quad T_{\mathrm{N}}=15 \mathrm{~ms}\right)$. Fig. 13 displays the measured closed loop transfer function of the position control (reference behaviour). The local and modal approach are compared as well as position control without additional active damping. In each case, the gains $K_{\mathrm{V}}$ of the position loops are tuned individually while keeping all maxima of the response functions close to $1(0 \mathrm{~dB})$.

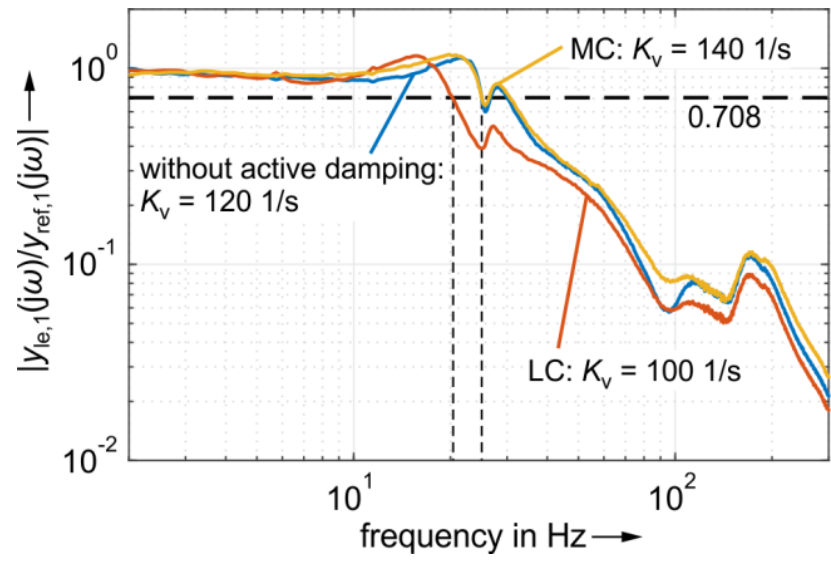

Fig. 13: Measured closed loop FRFs.

As a result of the experiment, the local damping controller leads to a reduced bandwidth compared to the contro without additional damping. A further increase of $K_{\mathrm{V}}$ for the local control approach would lead to a higher amplitude at approx. $20 \mathrm{~Hz}$ in Fig. 13, well above $0 \mathrm{~dB}$, and would mean an unwanted overshoot of the target position. This is due to the active dampers counteracting the movement of the linear drives. In the case of the modal control, there is no reduction of the bandwidth as the control bandwidth is increased by $25 \%$. The decoupling control allows each eigenmode to be treated individually. Therefore, the second, third and fourth mode, which are representing the target vibration modes, can be damped with high gains with no influence on the position control. Conversely, in the first modal control loop, which acts on the rigid body movement, a low gain value is used. The disturbance behaviour of the closed loops, which is measured with the impulse hammer, is depicted in Fig. 14. The local and modal damping controllers can be seen to achieve a similar behaviour. If the gains $k_{\mathrm{loc}, 1}$ and $k_{\mathrm{loc}, 2}$ would be reduced to realise a higher control bandwidth for the closed loop FRF depicted in Fig. 13, the disturbance response would deteriorate.

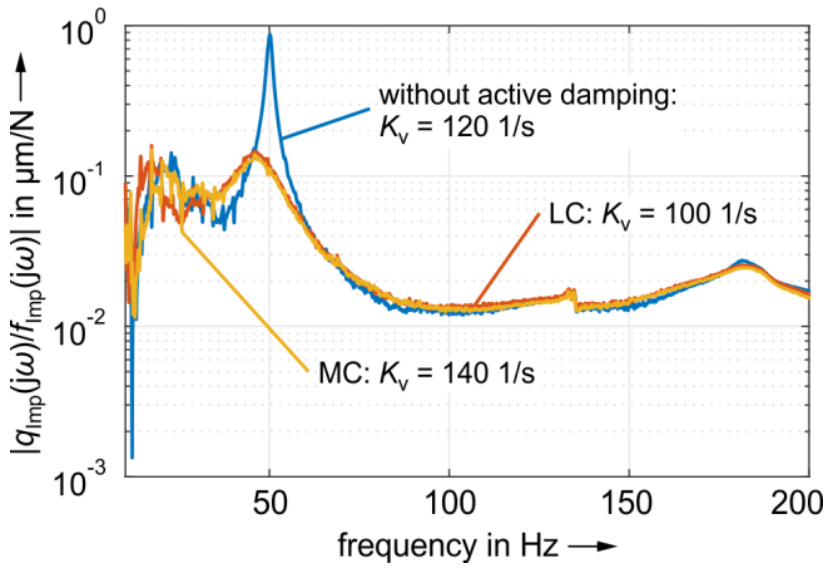

Fig. 14: Comparison of the measured FRFs: disturbance behaviour (excitation with impulse hammer).

\section{Comparison of the actuator forces}

The following analysis is intended to compare the interference between the control loops for both control strategies. The system is excited using ADD 2 and the resulting force amplitudes of the actuators are compared. This can be assumed as a disturbance due to a process force at the TCP. The resulting compensation forces of the actuators are measured and analysed in the frequency domain

Fig. 15 compares the magnitudes of the ADDs in the frequency domain for the local and modal control approach. The controller gains, specified in Tab. 1, are used. The local and modal control loops are tuned to achieve a similar damping behaviour at the frequencies of the controlled mode shapes. It can be seen that at a frequency of approx. $100 \mathrm{~Hz}$ the force amplitudes of the local controller are higher than the modal ones. This results from the fact that in the case of the modal approach the linear motors are used as well as the active dampers. The required forces are distributed between the linear motors and the damping actuators. This is a positive effect because it is possible to downsize the additional damping actuators when the modal control approach is applied. A closer look at the frequency at approx. $50 \mathrm{~Hz}$ shows that the amplitudes are almost the same. Since the linear drives have no influence on this mode, only the ADDs are able to affect it, both with modal and local control. 


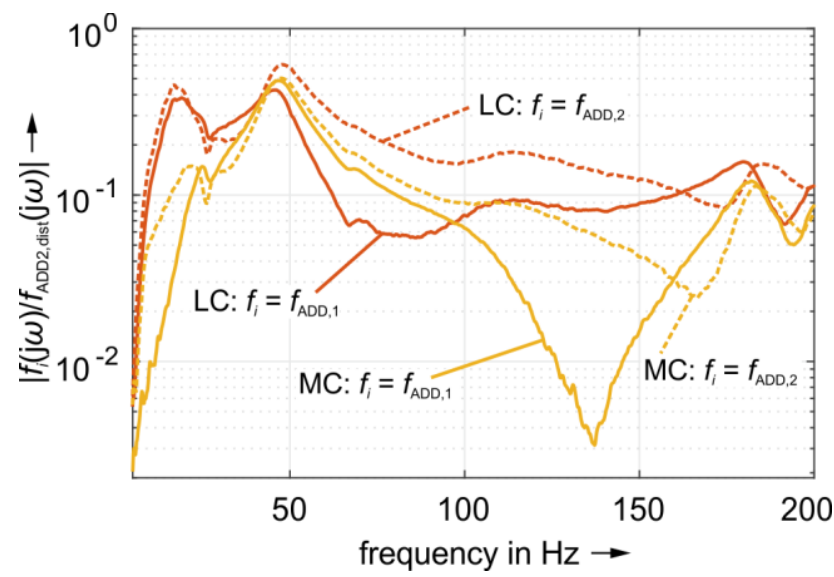

Fig. 15: Measured FRFs of the compensation forces resulting from the ADDs, (CPV active, excitation with $A D D$ 2).

In Fig. 16 the amplitudes of the linear motors are depicted, which are measured under the same conditions as the forces of the ADDs in Fig. 15. The curve characteristic of the compensation forces resulting from the linear motors of the modal control is almost the same as the case when no active damping is present (compare Fig. 16). Furthermore, it can be seen from Fig. 15 and 16 that in the frequency range below approx. $20 \mathrm{~Hz}$, the amplitudes of the local damping control are higher than the amplitudes of the modal control and the one without additional damping. Therefore, it seems that for the local approach, the forces of the linear motors counteract the forces of the ADDs in this frequency range. It fits to the fact that the different control loops disturb each other. In contrast, there is a negligible interference between the ADDs and the linear motors in this frequency range for the modal control. This is likely to have a positive effect on the command response.

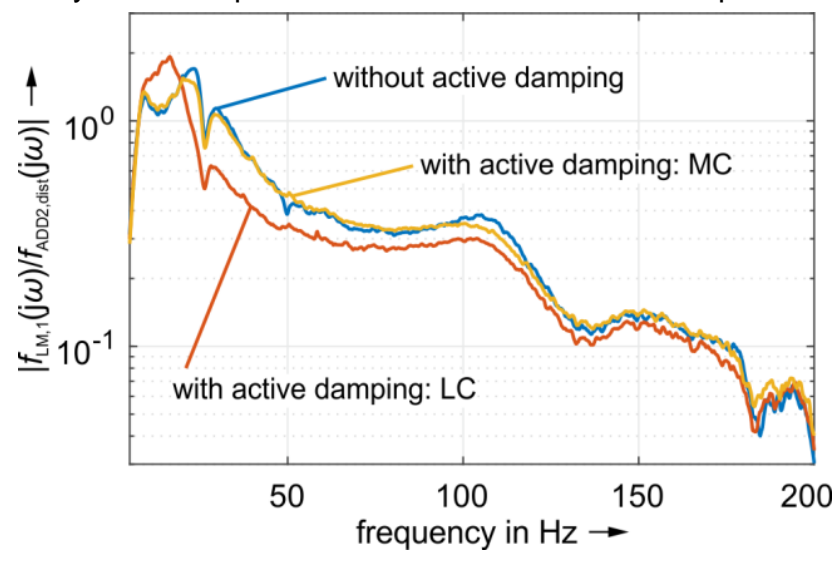

Fig. 16: Measured FRFs of the compensation forces resulting from linear motor 1 , (CPV active, excitation with $A D D 2)$.

\section{SUMMARY}

Usually, the actuators or drives of motion systems or machine tools are controlled independently of each other, without considering their coupling. In this paper, the modal control approach has been applied to consider the mechanical coupling. This has made it possible to control the eigenmodes individually and to damp the excited vibrations with all available actuators. This model-based control approach is particularly suitable for motion systems, such as machine tools, which have more drives or actuators than degrees of freedom of movement. Experimental results of the modal control of a gantry arrangement have been presented. With the implemented modal four-variable control, the control bandwidth of the position controllers was able to be increased and a similar disturbance behaviour at the TCP was achieved, compared to the local approach. Due to the increased control bandwidth, the dynamic path accuracy and thus the accuracy of the workpiece is improved. Furthermore, by applying the modal decoupling technique, the parameterisation of the controllers is simplified. The modal control approach is advantageous since all sensor signals are taken into account in each modal control loop. In addition, the modal forces required for vibration damping are specifically generated by all existing actuators

Since the eigenfrequencies of the system are not used directly to tune the proposed controllers, small changes of these values do not cause stability issues. However, when the parameters of the system, in particular the mode shapes, vary in a wider range, the advantages of the modal approach will disappear if the control parameters are not adapted. Therefore, the consideration of parameter variations of the dynamic behaviour of the system, especially by the variation of mode shapes due to different poses, will be the subject of future research.

\section{ACKNOWLEDGEMENTS}

This research was supported by a German Research Foundation (DFG) grant, received within the research project 'Fundamental analysis of the modal control applied to over-actuated machine tools' (IH 124/4-1), which is gratefully acknowledged. We would like to thank the Fraunhofer IWU - Machine Tool Department, in particular Dr. Bergmann and Mr. Wabner for providing technical equipment. The authors also thank Mr. Kretzschmar who supported us in carrying out experiments and $\mathrm{Mr}$. Holowenko who supported us with the data acquisition.

\section{REFERENCES}

[Balas 1978] Balas, M. J. Active control of flexible systems. Journal of Optimization Theory and Applications, 1978, Vol.25, No.3, pp. 415-436.

[Belyaev 2017] Belyaev, K. A., Polyanskiy, V. A., Smirnova, N. A. and Fedotov, A. V. Identification procedure in the modal control of a distributed elastic system. Physics and Mathematics. 2017, Vol.3, pp. 135-143.

[Braghin 2012] Braghin, F., Cinquemani, S., Resta, F. A new approach to the synthesis of modal control laws in active structural vibration control. Journal of Vibration and Control, Vol.19, No.2, pp.163-182.

[Brecher 2013a] Brecher, C., Gsell, S. and Lohse, W. Schwingungsdämpfung in Werkzeugmaschinen Lageistwertfilter zur Unterdrückung von Resonanzen im Lageregelkreis. wt Werkstattstechnik online, 2013, Vol.103, No.5, pp. 388-394.

[Brecher 2013b] Brecher, C., Baumler, S. and Brockmann, B. Avoiding chatter by means of active damping systems for machine tools. Journal of Machine Engineering, Vol.13, No.3, pp. 117-128.

[Dorf 2011] Dorf, R. C. and Bishop, R. H. Modern Control Systems, 2010, Pearson, ISBN 978-0-13-138310-4.

[Drossel 2016] Drossel, W.-G., Junker, T., Schmidt, S. and Pagel, K. Cost-efficient approach for impulse decoupled linear direct drives. Production Engineering, 2016, Vol.10, No.3, pp. 361-366. ISSN 1863-7353.

[Gazzulani 2012] Cazzulani, G., Resta, F., Ripamonti, F. and Zanzi, R. Negative derivative feedback for vibration 
control of flexible structures. Smart Materials and Structures, 2012, Vol.21, No.7, pp. 1-10.

[Gould 1966] Gould, L. A. and Murray-Lasso, M. A. On the Modal Control of Distributed Systems with Distributed Feedback. IEEE Transactions on Automatic Control, 1966, Vol.11, No.4, pp. 729-737.

[Großmann 2014]. Großmann, K., Müller, J., Merx, M., Peukert, C. Reduktion antriebsverursachter Schwingungen. Antriebstechnik / ant Journal. 2014, Vol.53, No.4, pp. 35-42.

[Garcia-Herreros 2013] Garcia-Herreros, I., Kestelyn, X., Comand, J., Coleman, R., Barre, P.-J. Model-based decoupling control method for dual-drive gantry stages: A case study with experimental validations. Control Engineering Practice. 2013, Vol.21, pp. 298-307

[Ihlenfeldt 2018] Ihlenfeldt, S., Müller, J., Merx, M. and Peukert, C. A Novel Concept for Highly Dynamic OverActuated Lightweight Machine Tools, In: Yan X., Bradley D., Moore P. ed., Reinventing Mechatronics: Proceedings of Mechatronics 2018, Glasgow, pp. 210-216.

[Ihlenfeldt 2019] Ihlenfeldt, S., Müller, J., Merx, M. and Peukert, C. Kinematically Coupled Force Compensation Experimental Results and Advanced Design for the 1DImplementation. Journal of Manufacturing and Materials Processing, 2019, Vol.3, No.1, pp. 1-13. ISSN 2504-4494

[Inman 2001] Inman, D. J Active modal control for smart structures. Philosophical Transactions of the Royal Society, 2001, Vol.359, pp. 205-219.

[Kroll 2011] Kroll, L., Blau, P., Wabner, M., Frieß, U., Eulitz J., Klärner, M. Lightweight components for energy-efficient machine tools. CIRP Journal of Manufacturing Science and Technology, 2011, Vol.4, No.2., pp. 148-160.

[Lopez de Lacalle 2009] Lopez de Lacalle, N., Lamikiz Mentxaka, A. Machine Tools for High Performance Machining. 2009, Springer-Verlag London.

[Löser 2018] Löser, M., Otto, A., Ihlenfeldt, S. and Radons, G. Chatter prediction for uncertain parameters, Advances in Manufacturing, 2018, Vol.6, No.3, pp. 310-333.

[Meirovitch 1983a] Meirovitch, L. and Baruh, H. Robustness of the independent modal-space control method. Journal of Guidance, Control, and Dynamics, 1983, Vol.6, No.1, pp. 20-25. ISSN 0731-5090.

[Meirovitch 1983b] Meirovitch, L., Baruh, H. and Oz, H. A comparison of control techniques for large flexible systems. Journal of Guidance, Control, and Dynamics, 1983, Vol.6, pp. 302-310.

[Meirovitch 1983c] Meirovitch, L. and Baruh, H. On the Problem of Observation Spillover in Self-Adjoint Distributed-Parameter Systems. Journal of Optimization Theory and Applications, 1983, Vol.39, No.2, pp. 269-291.

[Meirovitch 1985] Meirovitch, L. and Baruh, H. The implementation of modal filters for control of structures. Journal of Guidance, Control, and Dynamics, 1985, Vol.8, No.6, pp. 707-716.

[Meirovitch 1990] Meirovitch, L. Dynamics and Control of Structures. 1990, John Wiley \& Sons.
[Murray-Lasso 1965] Murray-Lasso, M. A. The modal analysis and synthesis of linear distributed control systems. M.I.T., Department of Electrical Engineering, 1965.

[Peukert 2017] Peukert, C., Merx, M., Müller, J. and Ihlenfeldt, S. Flexible coupling of drive and guide elements for parallel-driven feed axes to increase dynamics and accuracy of motion. Journal of Machine Engineering, 2017, Vol.17, No.2, pp. 77-89.

[Peukert 2019a] Peukert, C., Pöhlmann, P., Merx, M., Müller, J. and Ihlenfeldt, S. Investigation of Local and Modal Based Active Vibration Control Strategies on the Example of an Elastic System. Journal of Machine Engineering, 2019, Vol.19, No.2, pp. 32-45.

[Peukert 2019b] Peukert, C., Pöhlmann, P., Ihlenfeldt, S., Müller, J. and Merx, M. Modale Regelung von Werkzeugmaschinen - Anwendung der modalen Regelung für eine Gantry-Anordnung. wt Werkstattstechnik online, 2019, Vol.109, No.5, pp. 358-364.

[Porter 1972] Porter, B. and Crossley, R. Modal Control Theory And Applications. 1972, Taylor \& Francis LTD.

[Resta 2010] Resta, F., Ripamonti, F., Cazzulani, G. and Ferrari, M. Independent modal control for nonlinear flexible structures: An experimental test rig. Journal of Sound and Vibration. 2010, Vol.329, pp. 961-972.

[Rosenbrock 1962] Rosenbrock, H. H. Distinctive problems of process control. Chemical Engineering Progress, 1962, Vol.58, pp. 43-50.

[Schröder 2007] Schroeder, T., Krabbes, M. and Neugebauer, R. Reactive trajectory splitting function for machine tools with hierarchical drive structures. The International Journal of Advanced Manufacturing Technology. 2007, Vol.33, No.9-10, pp. 988-993.

[Serra 2013] Serra, M., Resta, F., Ripamonti and F. Dependent modal space control. Smart Materials and Structures, 2013, Vol.22, No.10, pp. 1-11.

[Simon 1968] Simon, J. D. and Mitter, S. K. A Theory of Modal Control. 1968, Information and Control. Vol.13, pp. 316-353.

[Skidmore 1985] Skidmore, G. R. Modal-space active damping of a beam-cable structure: theory and experiment. Journal of Sound and Vibration. 1985, Vol.101, No.2, pp. 149-160.

[Yang 2010] Yang, Y., Muñoa, J., Altintas, Y. Optimization of multiple tuned mass dampers to suppress machine tool chatter. International Journal of Machine Tools and Manufacture. 2010, Vol.50, No.9, pp. 834 - 842. ISSN 0890 6955.

[Wang 2002] Wang, D.-A. and Huang, Y.-M. Modal space vibration control of a beam by using the feedforward and feedback control loops. International Journal of Mechanical Sciences. 2002, Vol.44, No.1, pp. 1-19.

[Zhang 2014] Zhang, Q., Jin, J., Zhang, J. Zhao, C. Active Vibration Suppression of a $3-D O F$ Flexible Parallel Manipulator Using Efficient Modal Control. Shock and Vibration, 2014, pp. 1-10. 